\title{
Effect of intra-operative hyperoxia on the incidence of surgical site infections: a meta-analysis
}

\author{
Barak Cohen, Yehoshua N. Schacham, Sanchit Ahuja, Ming H. Hung, \\ Alicia Barkley, Daniel I. Sessler, Kurt Ruetzler
}

\section{Outcomes Research Department, Anesthesiology Institute, Cleveland Clinic, Cleveland, United States}

Background: Whether supplemental intraoperative oxygen reduces surgical site infections remains unclear.

Recent recommendations from the World Health Organization and Center for Disease Control to routinely use high inspired oxygen concentrations to reduce infection risk have been widely criticized. We therefore performed a meta-analysis to evaluate the influence of inspired oxygen on infection risk, including a recent large trial.

Methods: A systematic literature search was performed. Primary analysis included all eligible trials. Sensitivity analyses distinguished studies of colorectal and non-colorectal surgeries, and excluded studies with high risk of bias.

Results: The primary analysis included 26 trials $(\mathrm{N}=14,710)$. The $R R$ $[95 \% \mathrm{Cl}]$ for wound infection was 0.81 [0.70, 0.94] in the high vs. low inspired oxygen groups [Fig.1]. The effect remained significant in colorectal patients $(\mathrm{N}=10,469), 0.79[0.66,0.96]$, but not in other patients $(\mathrm{N}=4,241)$, $0.86[0.69,1.09]$.

When restricting the analysis to studies with low risk of bias, either by strict inclusion criteria $(\mathrm{N}=5,047)$ or by researchers' judgment $(\mathrm{N}=12,547)$ [Fig.2], no significant benefit remained: 0.84 [0.67, 1.06] and $0.89[0.76,1.05]$, respectively.

Conclusions: When considering all available data, intraoperative hyperoxia reduced wound infection incidence. No significant benefit remained when analysis was restricted to low-bias studies.

Meta-analysis of the most reliable studies does not suggest that supplemental oxygen substantively reduces wound infection risk.

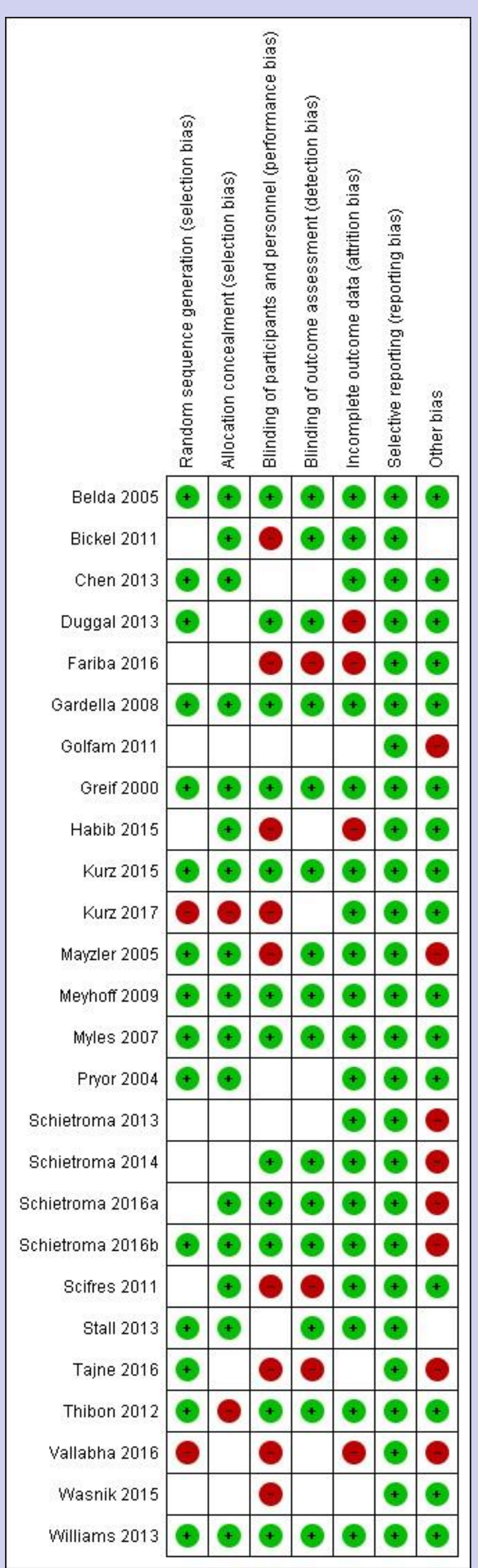

\begin{tabular}{|c|c|c|c|c|c|}
\hline \multicolumn{6}{|c|}{ Sensitivity Analysis - low risk of bias } \\
\hline Author(s) and Year & $\begin{array}{ll}\text { No. ssil } \\
\text { High Fio, }\end{array}$ & 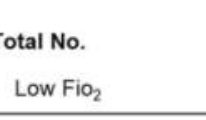 & & Weight & Relative Risk $[95 \% \mathrm{Cl}]$ \\
\hline Greif 2000 & $13 / 250$ & $28 / 250$ & $\longmapsto$ & $4.87 \%$ & $0.46[0.25,0.88]$ \\
\hline Pryor 2004 & 20180 & $9 / 80$ & & $3.95 \%$ & $2.22[1.08,4.58]$ \\
\hline Belda 2005 & 22/148 & $35 / 143$ & $\mapsto$ & $7.28 \%$ & $0.61[0.38,0.98]$ \\
\hline Myles 2007 & 771997 & 106/1015 & 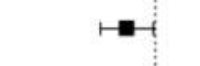 & $13.07 \%$ & $0.74[0.56,0.98]$ \\
\hline Gardella 2008 & $17 / 69$ & $10 / 74$ & & $4.08 \%$ & $1.82[0.90,3.70]$ \\
\hline Meyhoff 2009 & $131 / 685$ & $141 / 701$ & 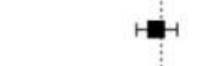 & $15.78 \%$ & $0.95[0.77,1.18]$ \\
\hline Thibon 2012 & $15 / 226$ & 15/208 & $\longmapsto$ & $4.26 \%$ & $0.92[0.46,1.84]$ \\
\hline Chen 2013 & $5 / 30$ & $19 / 61$ & - & $2.81 \%$ & $0.54[0.22,1.29]$ \\
\hline Duggal 2013 & $34 / 416$ & $32 / 415$ & $\mapsto$ & $7.65 \%$ & $1.06[0.67,1.68]$ \\
\hline Stall 2013 & 14/119 & 19/116 & $\longmapsto$ & $4.79 \%$ & $0.72[0.38,1.36]$ \\
\hline Williams 2013 & $10 / 77$ & $12 / 83$ & $\longmapsto$ & $3.48 \%$ & $0.90[0.41,1.96]$ \\
\hline Kurz 2015 & $45 / 285$ & $42 / 270$ & $\mapsto$ & $9.56 \%$ & $1.02[0.69,1.49]$ \\
\hline Kurz 2017 & 295/2896 & $314 / 2853$ & - & $18.42 \%$ & $0.93[0.80,1.08]$ \\
\hline \multicolumn{3}{|c|}{$\operatorname{RE} \operatorname{Model}\left(\mathrm{Q}=21.5, \mathrm{df}=12, \mathrm{P}=0.043 ; \mathrm{I}^{2}=44.2 \%\right)$} & $\bullet$ & $100.00 \%$ & $0.89[0.76,1.05]$ \\
\hline & & & 0.25 & & \\
\hline
\end{tabular}

